\title{
A note on multiple comparisons as an ANOVA problem
}

\author{
JOHN GAITO and JOSÉ N. NOBREGA \\ York University, Downsview, Ontario M3J IP3, Canada
}

\begin{abstract}
A short discussion of multiple-comparison procedures is presented. The types of procedures used by experimental psychologists as reported in one general experimental psychology journal during a 2-year period (1978-1979) were determined. A variety of such procedures were employed. It is suggested that any set of multiple comparisons desired by a researcher can be obtained quickly and efficiently by multiple $F$ tests within the ANOVA framework.
\end{abstract}

When a researcher uses ANOVA procedures and there are only two levels of the dimension or factor of concern, the $F$ test usually provides all of the information necessary for conclusions and inferences. However, if there are more than two levels in the dimension, the $\mathrm{F}$ test provides incomplete information. In such a case, most researchers will stop if the $F$ test results in a nonrejection of the null hypothesis $\left(\mathrm{H}_{0}\right)$. If rejection of $\mathrm{H}_{0}$ occurs, however, the researcher must resort to some type of multiple-comparison procedure to gain more complete information concerning the number of population distributions involved in the dimension (Kirk, 1968; Winer, 1971).

The problem of multiple-comparison procedures seems to be one of the most puzzling aspects in psychological statistics for many users of statistical methods. This point is illustrated in the questions posed by students and faculty seeking advice on statistical research problems. There is a bewildering array of multiplecomparison procedures, each one having features that are often only slightly different from the others. Thus, the person who has little or moderate experience in statistics can be confused by the number of multiplecomparison procedures available.

It is amazing that so much effort has been devoted to developing new procedures for handling the multiplecomparison problem when there has been a simple solution for many years. Any type of comparison desired can be effected easily and efficiently within the ANOVA framework using multiple $F$ tests (Gaito, 1978).

We decided to assess the actual multiple-comparison practices followed by experimental psychologists. We ascertained the procedures used by contributors to a specific psychological journal (Bulletin of the Psychonomic Society) over a period of 2 years (19781979). This journal was chosen for a number of reasons: (1) It is a periodical used by many experimental psychologists, who make frequent use of ANOVA and multiple-comparison procedures. (2) Almost every paper makes use of statistical tests of null hypotheses. (3) Papers are limited to four or fewer pages. Thus, many papers are contained within each edition, and the size of the sample could be reasonably large within the 2-year period. (4) The topics covered are very general within experimental psychology, thus providing some degree of generality across experimental psychology topics.

A total of 507 articles were examined from the 2-year period. Of these papers, 88 had no statistical analyses of $\mathrm{H}_{0}$ and 8 did not specify the actual statistical procedures used. Of the remaining 411 papers, 314 used ANOVA (76.4\%). It is interesting to note that in 15 of the other 97 papers, multiple t tests without an omnibus $\mathrm{F}$ test were employed. In the remaining 314 papers using ANOVA, there were no multiple comparisons involved in 108 .

The results for the 206 papers that used ANOVA and a multiple-comparison procedure are shown in Table 1. The ones used most frequently were multiple $t$ tests, ANOVA multiple $F$ tests, and the Newman-Keuls procedure. ${ }^{1}$ A variety of other procedures were used less frequently, including nonparametric procedures. Although the practices of other psychologists may vary somewhat from those of experimental psychologists, there is no reason to suppose that the discrepancy would be great.

Thus, it seems that psychologists use a variety of multiple-comparisons procedures unnecessarily, for

Table 1

Multiple-Comparison Procedures Used in 206 Articles

\begin{tabular}{lcc}
\hline \multicolumn{1}{c}{ Procedure } & Frequency & Percentage \\
\hline Multiple t Tests & 51 & 24.8 \\
ANOVA Multiple F Tests & $50^{*}$ & 24.3 \\
Newman-Keuls & 43 & 20.9 \\
Tukey & 21 & 10.2 \\
Duncan & 17 & 8.3 \\
Scheffé & 7 & 3.4 \\
$\chi^{2}$ & 6 & 2.9 \\
Mann-Whitney & 3 & 1.5 \\
Dunnett & 2 & 1.0 \\
Cicchetti Test & 1 & .5 \\
Not Specified & 5 & 2.4 \\
\hline
\end{tabular}

${ }^{*}$ Six of these were tests of trends. 
any results provided by other multiple-comparison procedures can also be provided by ANOVA F tests. It is unfortunate that so much attention has been devoted to developing new multiple-comparison procedures and that so much time and effort is wasted in determining which procedure to use and in understanding the procedure. Psychologists could be using this time and effort on important substantive research or theoretical problems of a nonstatistical nature.

The various multiple-comparison procedures have focused attention almost exclusively on the Type I error rate (false positives). Such exclusive emphasis on this error rate has been unfortunate. In many cases (e.g., initial experimentation in a specific area), the Type II error rate (false negatives) may be more important. False positive results would be recognized as errors with the repeated experimentation involved in a systematic research program. However, false negative results would be lost because researchers usually discard variables involved in results that have not been found to be statistically significant. In any event, either error rate can be handled effectively with ANOVA $F$ tests. For example, the Type I error rate can be controlled by adjusting the $\alpha$ levels to the specific value that the researcher desires (Gaito, 1978), thereby modifying Type II error rates, as well.

Not only is the ANOVA F test procedure for comparisons a simple one, but it is also entirely possible that some of the multiple-comparison procedures may be affected adversely more than are ANOVA F tests when violations from assumptions occur. The ANOVA $F$ tests are sensitive to the violation of the independence assumption (Gaito, 1973), but they are relatively insensitive to the assumptions of homogeneity of variance and normality of distribution (Gaito, 1959). Such may not be the case with some of the other multiple-comparison procedures.

From the above comments, it is obvious that we would recommend that psychologists disregard the various non-ANOVA multiple-comparison procedures and use ANOVA methods in every case. The usual strategy we follow is to perform an overall or omnibus $F$ test evaluating the differences in means between the various groups. If $\mathrm{H}_{0}$ is not rejected, no further $\mathrm{F}$ tests are attempted. However, if $\mathrm{H}_{0}$ is rejected, specific comparisons can then be attempted. These can be simple comparisons (one group vs. another group) or complex comparisons (more than one group vs. one or more than one other group). Obviously, the specific comparisons attempted should be those that are meaningful in the research effort.

Likewise, the comparisons can be orthogonal or nonorthogonal (Gaito, 1978). With orthogonal comparisons, the number of separate comparisons cannot be greater than the degrees of freedom for the main effect; the sum of the sums of squares and degrees of freedom involved in each $F$ test for each comparison equal the sums of squares and degrees of freedom for the main effect. However, nonorthogonal comparisons are not restricted in this fashion; many comparisons can be performed, for example, any simple or complex comparisons as handled by the multiple $t$, Newman-Keuls, Tukey, Scheffé, Dunnett, and other multiple-comparison procedures.

\section{REFERENCES}

GAITo, J. Non-parametric methods in psychological research. Psychological Reports, 1959, 5, 115-125.

Gaito, J. Repeated measurements designs and tests of null hypotheses. Educational and Psychological Measurement, 1973, 33, 69-75.

GAITo, J. Multiple comparisons within ANOVA using orthogonal and nonorthogonal components. Educational and Psychological Measurement, 1978, 38, 901-904.

KIRK, R. E. Experimental design: Procedures for the behavioral sciences. Belmont, Calif: Brooks/Cole, 1968.

Winer, B. J. Statistical principles in experimental design. New York: McGraw-Hill, 1971.

\section{NOTE}

1. Following an omnibus $F$ test, multiple $t$ tests and multiple $F$ tests are quite similar. However, there is an important distinction. Multiple $t$ tests make use of the data only for the two groups of concern in each test. Multiple $F$ tests involve a subdivision of the main effect of concern; the error term is the same as that for the omnibus $F$ test, providing greater degrees of freedom for the denominator and leading to greater power.

(Received for publication February 16, 1981.) 